\title{
Abnormal deposits of chromium in the pathological human brain
}

\author{
SERGE DUCKETT \\ From the Division of Neuropathology, Department of Neurology, Jefferson Medical College of the Thomas \\ Jefferson University, Philadelphia, USA
}

SUMMARY Three patients presented with encephalopathies: an undiagnosed degenerative disease of the brain, a degenerative cerebral disease in a patient with a myeloma but without a myelomatous deposit in the CNS and a malignant astrocytoma. Perivascular pallidal deposits (vascular siderosis) containing chromium, phosphorus and calcium plus sometimes traces of other elements were present in the three cases. Such deposits were present in the pallidal parenchyma and around vessels in the cerebellum in one case. Calcium and phosphorus are always present in any CNS calcification but the presence of chromium has not been reported. Chromium and its compounds (ingested, injected or inhaled) are toxic to humans and animals in trace doses. Approximately 900 cases of chromium intoxication have been reported and usually have had dermatological or pulmonary lesions (including cancer) but there is no report of involvement of the CNS. Sublethal doses of chromium nitrate injected intraperitoneally in rats and rabbits results in the presence of chromium in the brain. A thorough investigation was made to find the source of the chromium in these patients. Chromium was found to be present in trace amounts in the radiological contrast agents administered to these patients and in the $\mathrm{KCl}$ replacement solution and in mylanta, an antacid, given to one case. The evidence that chromium induced pathological changes in these three brains is circumstantial but shows that chromium can penetrate the human brain. This study indicates that vascular siderosis found in the brains of the majority of middle-aged and elderly humans is not simply an anecdotal pathological curiosity, but that it can serve as a route of entry for toxico products into the brain.

No report has been found in the medical literature of a case of chromium induced pathology of the human central nervous system. We now describe abnormal deposits (calcifications) containing chromium in three pathological human brains. These cases were examined in great detail: their profession, life style, geographical location, past family and physical histories. Every hospital product they received for therapeutic purposes was analysed. Complete necropsies, general and neuropathological histological examinations, and electron microprobe $x$ ray analysis were done.

\section{Methods of preparation and study of tissue}

Tissue specimens from the central and peripheral nervous

Address for reprint requests: Serge Duckett, MD, Jefferson Medical College, Department of Neurology, 1025 Walnut Street, Philadelphia, PA 19107, USA.

Received 25 September 1985 and in revised form 14 June 1985. Accepted 24 June 1985 systems, lung, liver, kidney, thyroid, adrenal gland, muscle, pancreas, stomach, small and large intestine of the three cases discussed here were fixed in $10 \%$ formol for at least one month and embedded in paraffin, cut in sections $7 \mu \mathrm{m}$ thick, stained with haematoxylin-eosin, haematoxylin van Gieson, Nissl, PAS, luxol fast blue and Holmes silver stain. Formol fixed specimens were further fixed in $2.5 \%$ gluteraldehyde, buffered with $0.1 \mathrm{~N}$ sodium phosphate, postfixed with $1 \%$ osmium tetroxide, washed, dehydrated, embedded in Spurr resin medium, cut and stained with $1 \%$ lead citrate and $1 \%$ uranyl acetate.

Tissue was fixed in $10 \%$ formol for 8 to 14 months before preparation for the microanalytical studies reported here The tissue was washed for $\mathbf{4 8}$ hours in distilled water, frozen with liquid nitrogen, cut in sections 7 to $15 \mu \mathrm{m}$ thick and mounted on Teflon plastic slides. The identity of an element in a tissue or in a cell is determined by the bombardment of the area with electrons, thus producing $x$ rays of a specific wavelength which can be measured with a crystal using Bragg's law. The electron microprobe analysis was done with a wavelength dispersive $x$ ray spectrometer attached to a light and electron microsope, the Camebax (Stamford, Conn). Details of the instrumentation, methods and inter- 
pretation have recently been published. ${ }^{1}$ In this study the wavelengths, crystals and crystal angles, used to identify the principal elements were for phosphorus K @ 1-2 (1); 6.158

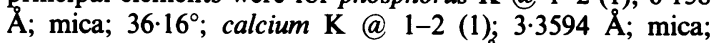
$19.50^{\circ}$; chromium K@ $@ 1-2$ (1); $2.291 \AA$; lithium fluoride (200); 69.36 ; iron K@ 1-2 (1); $1.9735 \AA$; mica; $11.20^{\circ}$; iodine L 1 (1); $3 \cdot 14860 \AA$; lithium fluoride (200); 102.8. The diameter of the area excited by the beam was $0.5 \mu \mathrm{m}$; the electron beam current was $2 \times 10-7 \mathrm{amp}$; the accelerating voltage $15 \mathrm{kV}$.

\section{Clinical and pathological data}

Case 1 A 73-year-old hypertensive lady treated successfully for hypertension during the past few years broke her right leg in October 1980. A cast was placed on it and removed at the end of December 1980. She felt well for a few days but a week later she began to have pain in her back and legs which increased in severity during the next two weeks. She was hospitalised on 20 January 1981 when it was concluded that there was a fracture at T12. Myelography was performed on 28 January for which she received $25 \mathrm{ml}$ of iophendylate. She was transferred the following day to the Jefferson Medical College. She had severe pain in the back, had difficulty moving her legs and had lost control of bladder and faeces; she was alert, pleasant, concerned and knowledgeable. Radiography revealed a mass in the body of T12 vertebra protruding into the cord, possibly the result of a pathological fracture or metastasis. On 4 February, 1981,

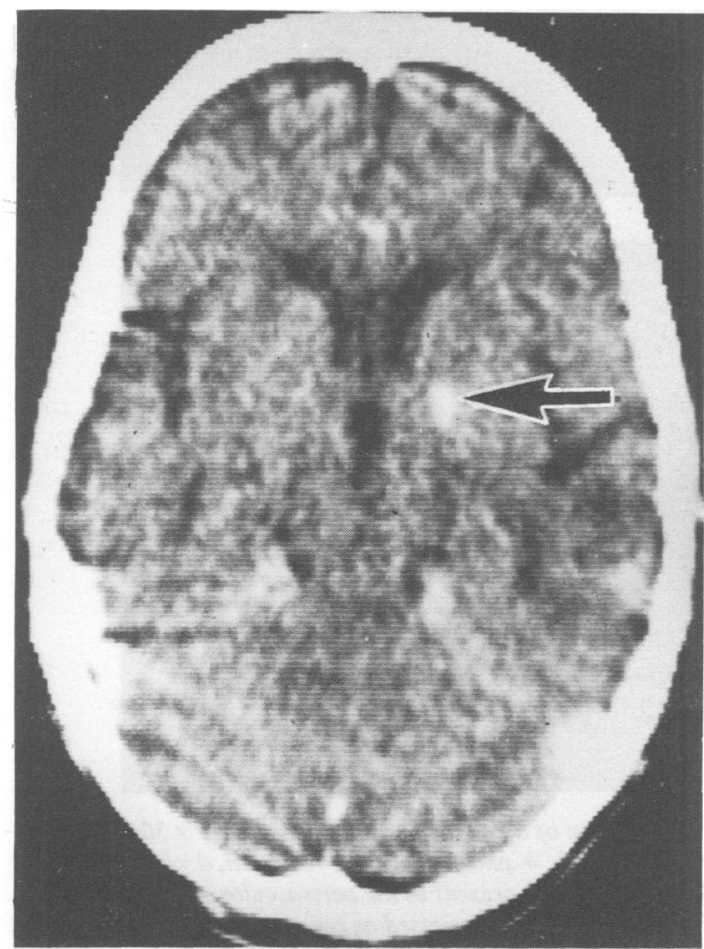

Fig 1 CT scan of the head of case 1. Note the pallidal calcification (arrow). she had a myelogram with $27 \mathrm{ml}$ iophendylate and an hour later there were "hallucinations, extreme agitation, confusion, self-mutilation and constant screaming." The psychiatric diagnosis was senile psychosis. No member of the family, nursing or medical staff had ever previously noted signs of mental disorder. Later that day she developed a volvulus and a variety of medical problems such as renal azotaemia, leucocytosis and thrombocytopenia. The dementia continued and varied in intensity but she improved physically. On 13 February a CT scan was done (with $12 \mathrm{ml}$ of the contrasting medium Conray (iothalamate meglumine). Diffuse brain atrophy and basal ganglia calcification on the right side were noted (fig 1). On 5 March, 1981 she was operated on and a lumbar protrusion was found to contain necrotic tissue but no tumour. The patient died the next day of post-operative complications. Gross examination of the brain confirmed the scan findings, namely frontal cortical atrophy, dilated ventricles, and calcification in the basal ganglia. The following tissues were examined: cervical and thoracic cord, medulla oblongata, pons, midbrain, diencephalon, basal ganglia, and representative areas from all lobes of the brain, the cerebellum and its nuclei, meninges and nerves from the cauda equina. There were senile plaques in the cerebral cortex in all lobes, and few neurofibrillary tangles. The cytoplasm of numerous neurons and glial cells was distended by lipofusion. There was demyelination in various lobes with gitter cells, in areas where a great many amyloid bodies were present. There was widespread distribution of hyperplastic and hyaline arteriolar sclerosis. Deposits were also present in the parenchyma particularly in the basal ganglia and dispersed in the rest of the brain. Calcified neurons were present in the basal ganglia. Many of the vessels in the globus pallidus and in the cerebellum, contained so-called calcifications or deposits in the wall and around the vessels (vascular siderosis) (fig 2). Electron microscopy examination revealed myelin bodies in some neurons. Microprobe examination of the various deposits revealed the presence of chromium, phosphorus, calcium, sometimes iron and rarely traces of iodine. The elements were present in the media of the small arteries (fig 2); chromium was also present in the perivascular space, sometimes associated with demyelination: evidence that it had crossed the wall of the vessel. Examination with the electron microscope showed that the intra- and perivascular deposits containing chromium and the other elements were composed of amorphous material woven in a fibrous matrix (fig 3 ).

The following organs and tissues were examined: kidneys, lungs, liver, thyroid, small and large intestines, muscle, adrenal gland, pancreas. There was tubular necrosis in the kidney, and pancreatitis. The principal neuropathological findings were: (1) generalised cortical atrophy, (2) hydrocephalus ex vacuo, (3) senile plaques throughout the cerebral cortex, (4) widespread diffuse demyelination with gitter cells, (5) cell loss in the cortex, (6) deposits in and around small blood vessels in the basal ganglia (vascular siderosis) and cerebellum, and in the parenchyma, containing chromium, phosphorus, calcium and traces of iron and iodine, (7) many amyloid bodies in the white matter indicative of fibrous astrocytic pathology, (8) arteriolar sclerosis, (9) myelin bodies in neurons. The final diagnosis was a cerebral degenerative disease of unknown cause.

Case 2 At the age of $63 \mathrm{yr}$, in early 1977, this lady was 
diagnosed as having multiple myeloma. Her marrow showed two thirds replacement with myeloma cells. Presenting clinical signs at the time were horizontal diplopia, left eye pain and swelling. The skull radiographs showed multiple lithic lesions and destruction of the sellar floor with soft tissue masses in the ethmoid sinuses and destruction of the base of the sphenoid. She received radiation treatment to the eye, which resulted in better ocular movements. In November of 1977, she had a hip operation. On 26 February, 1978, she returned with the complaint of pain in her left arm, which was broken and contained myeloma cells. A CT scan (with $12 \mathrm{ml}$ Conray) was not informative. The information booklet packaged with Conray states "A definite risk exists in the use of intravascular contrast agents in patients who are known to have myeloma. In such instances anuria has developed resulting in progressive uraemia, renal failure and eventually death". The patient died the next day. Necropsy and histopathological examination confirmed the diagnosis of myeloma, with deposits in different areas of the body. Gross examination of the brain showed cortical atrophy over the frontal region. Sections of the frontal cortex, occipital cortex, basal ganglia, brainstem and cerebellum, optic nerve, retro-orbital fat, peripheral nerves and skeletal muscles were examined. There was calcification in the arterioles in the globus pallidus, and in the surrounding regions which contained chromium, phosphorus, calcium and iron. No myeloma deposits were found in the CNS. Pallor of myelin was observed in the optic nerve. The neuropathological findings were: (1) frontal cortical atrophy, (2) chromium in the vascular pallidal "calcifications", (3) possible optic nerve demyelination. The final diagnosis was metabolic encephalopathy.
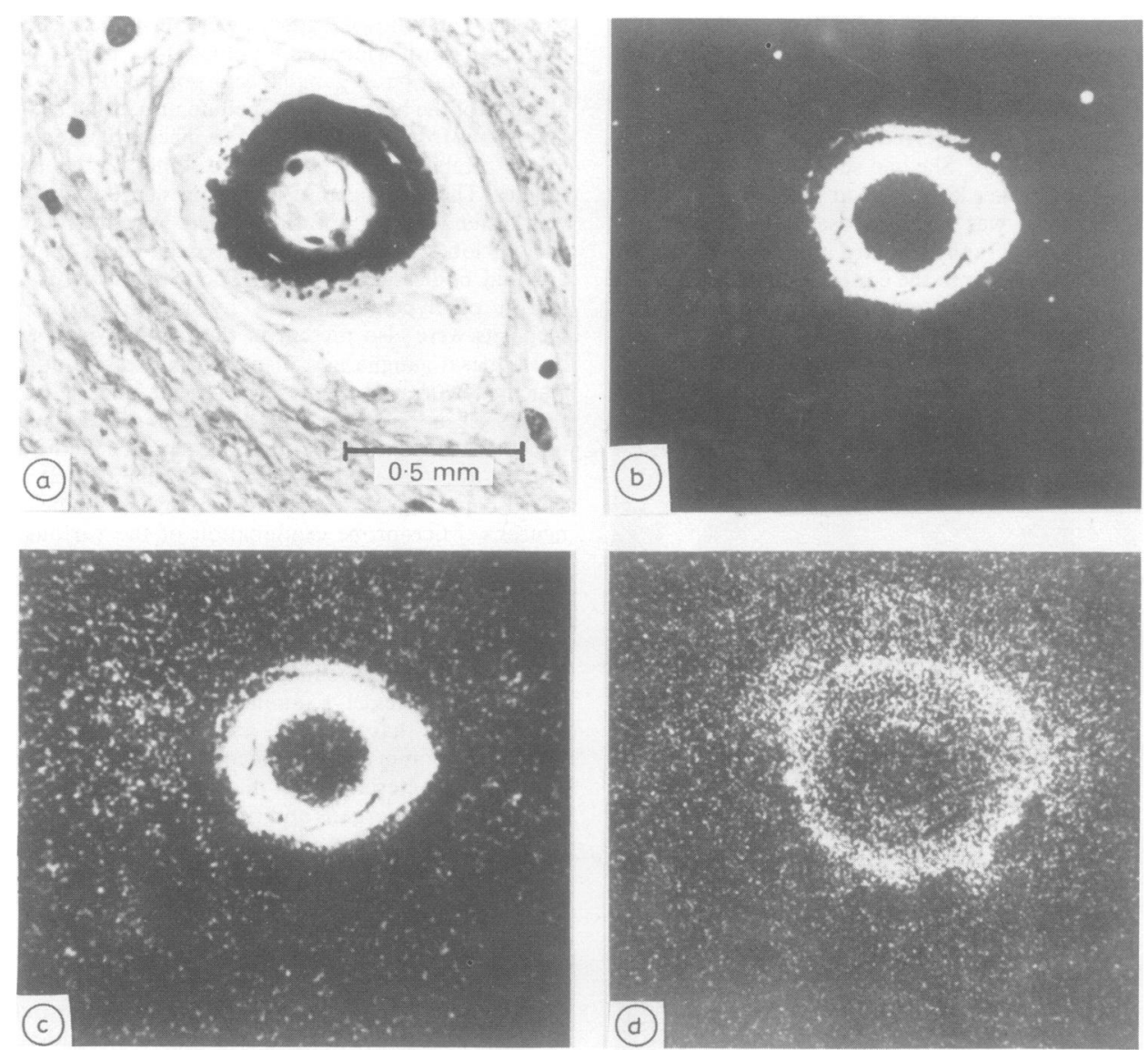

Fig 2 Photograph a is a haematoxylin and eosin stained preparation of a calcified pallidal arteriole $(\times 50)$. Each of the other photographs shows the distribution of one element $(b=$ phosphorus, $c=$ calcium, $d=$ chromium) as revealed by an electron microprobe. Note that chromium is present in the perivascular area. The identification is based on the number of photons emitted by an element, expressed as counts per second or per minute, which must be significantly more than that emitted by the background. ${ }^{1}$ Iron and iodine were sometimes detected in trace quantities in the calcified vessels. The graph (e, next page) illustrates the

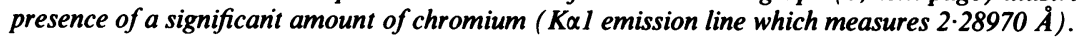




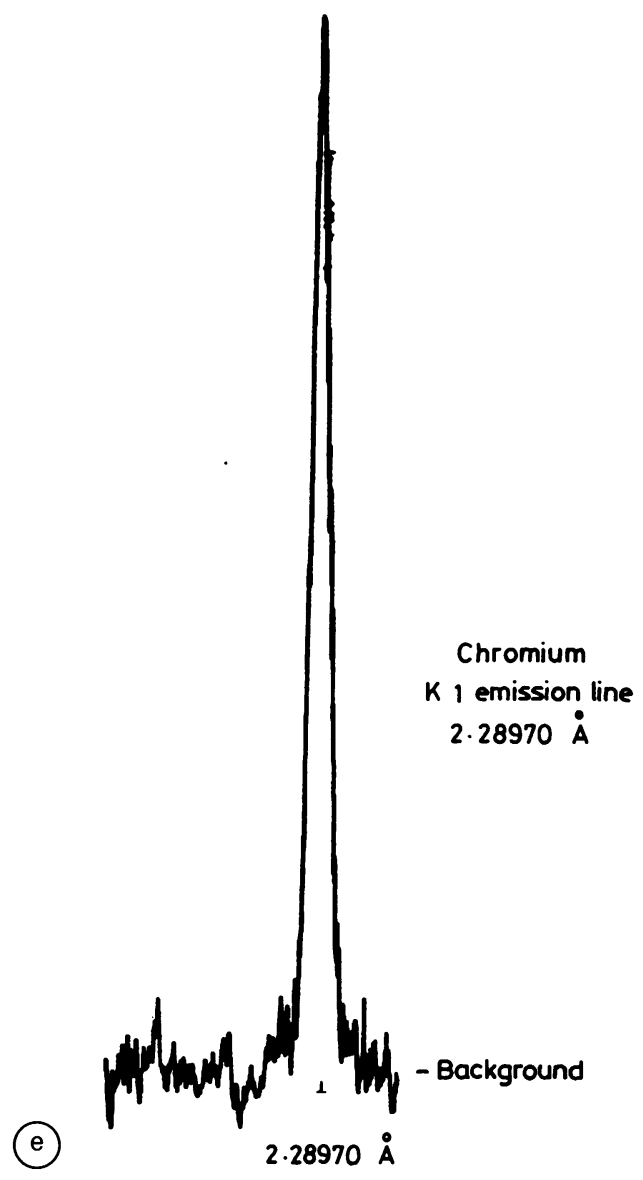

Case 3 The patient was a 51-year-old male, a salesman. For three weeks prior to hospitalisation (16 March, 1981) he presented with inappropriate behaviour, forgetfulness and drowsiness and was involved in two car accidents with bodily injuries. He was transferred to the Jefferson Hospital a few days later where an arteriogram (with an IV injection of $20 \mathrm{ml}$ Hypaque (diatrizoate meglumine) showed a large vascular neoplasm of the left front-temporal corpus callosum. A CT scan (with $12 \mathrm{ml}$ Conray) confirmed the presence of the tumour. He was operated upon on $30 \mathrm{March}$ and died on 6 April, 1981. The tumour was diagnosed as a glioblastoma multiforme. Tissue samples were taken from all the main somatic organs, the brainstem, cerebellum, diencephalon, basal ganglia and all cerebral lobes. The glioblastoma was present in the left temporal lobe invading surrounding areas of the brain. In the areas around the tumour there were petechiae, inflammatory cells, some perivascular inflammatory cell concentration and destruction of tissues. There were mineral deposits in the media of the small arteries in the globus which contained chromium, phosphorus, calcium. There was no chromium in the tumour. The diagnosis was malignant astrocytic tumour, and vascular siderosis with chromium in the deposits in the vascular wall.

\section{Biochemical analysis}

The quantitation of chromium in hospital medication and in radiology contrast medium was done with an induced coupled plasma (ICP) quantometer and recorded as parts per million (ppm). Conray (iothalamate meglumine) and Hypaque (diatrizoate meglumine) each contain chromium $0.2 \mathrm{ppm}$; Pantopaque (iophendylate) contains $0.8794 \mathrm{ppm}$ chromium. All three cases received Conray shortly before death; case 1 also had Pantopaque and case 2 had Hypaque. The amount of chromium injected into these patients is well below the lethal dosage for mammals. ${ }^{2}$ Between 28 January and 24 February, case 1 received IV potassium chloride (which contains 0.06 ppm chromium) every other day and Mylanta (Stuart Pharmaceuticals, Wilmington, Del) (chromium 3 ppm) on three occasions for a total of about $3 \mathrm{mg}$ chromium. Unfixed neural tissue of these cases was not available for quantitative studies.

\section{Discussion}

Abnormal perivascular and parenchymal mineral deposits which contained chromium were present in these three brains. The source of chromium may have been the radiological contrast substances injected into these patients shortly before death. It is evident that it entered the brain by way of the pathological pallidal

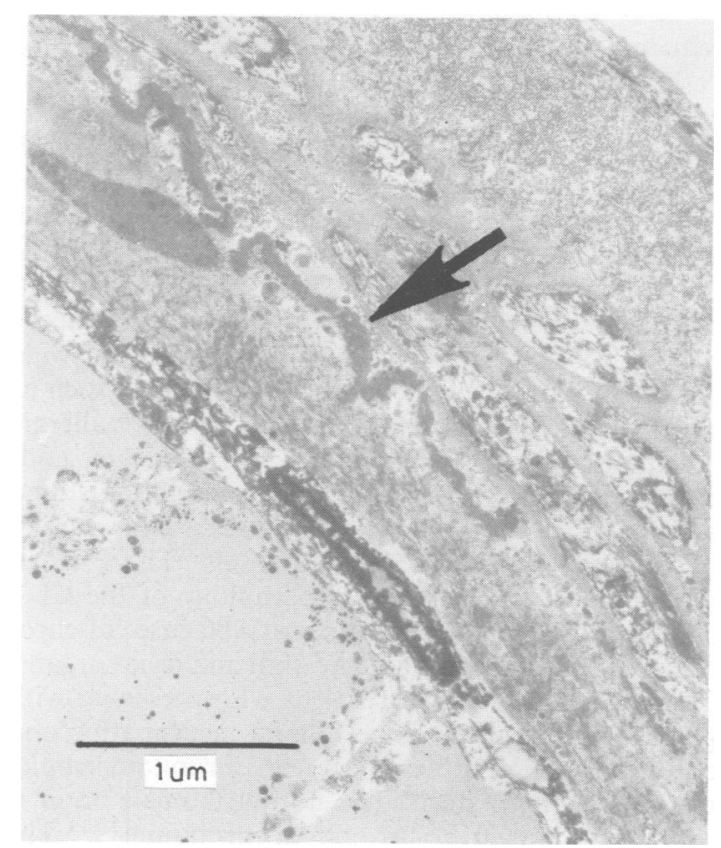

Fig 3 Electron micrograph of a portion of a pallidal calcified vessel. The elements are localised in the fibrous amorphous mass indicated by the arrow. 
blood vessels. The role of chromium in the death of patient 2 is difficult to assess. We will review briefly here the following: vascular siderosis, the biological role of chromium and the neurotoxicity of radiological contrast agents.

(1) Vascular siderosis This has been observed in the brains of the vast majority of middle aged and elderly humans seen at necropsy for nearly a century. ${ }^{3}$ Its cause is unknown but the studies of Alexander and Putnam ${ }^{4}$ may provide the answer. They noted that the two most vulnerable cerebral structures, the globus pallidus and the hippocampus, are both supplied by the anterior choroidal artery. They proposed that any artery's susceptibility to pathological damage could be expressed by the ratio of length over diameter. Thus the anterior choroidal artery is the artery most susceptible to damage in the brain; it has the longest free subarachnoid course of any artery of similar diameter and its pallidal branch has to bend within the brain substance to reach the main part of the globus pallidus. This is one explanation. Could there be biochemical changes within the globus pallidus that effect the vessels locally? Can chromium cause vascular pathology namely vascular siderosis? This question has not been examined. Denny-Brown ${ }^{5}$ suggested that pallidal pathology such as seen in Parkinson's disease may result from a defect in the vascular permeabilities of the blood barrier in the globus pallidus to metallic ions. This opinion was subsequently supported by our electron probe studies of vascular siderosis associated with Parkinson's disease. $^{3}$

(2) Chromium This has atomic number 24 with oxidation states chiefly $+2,+3,+6$. It occurs naturally as ferrous chromite. All chromium compounds whether ingested, injected or inhaled, are toxic to humans and animals in small doses. ${ }^{2}$ Chromium is an essential nutrient which plays a key role in glucose metabolism. ${ }^{6}$ Chromium derivatives cause a wide variety of pathological conditions in humans such as skin ulcers, acute irritative dermatitis, allergic eczematous dermatitis, ulcerated nasal septum, nodular pneumoconiosis, liver disease, bronchitis, asthma and lung cancer. ${ }^{78}$ Chromium causes a wide variety of tumours in experimental animals. There is no report of chromium-induced pathology of the CNS although there are reported nearly 900 cases of chromium intoxication of lung and skin and other organs. There is a report of one patient who presented with glucose intolerance, a peripheral neuropathy and chromium deficiency reversed with chromium supplementation in the diet. ${ }^{9}$ The normal human brain is said to contain 0.01 to $0.1 \mathrm{ppm}$ of chromium. ${ }^{1011}$ The brains of our patients were contaminated by the presence of chrome $(0.2 \mathrm{ppm})$ in the formol fixative.

None of the three patients described here had any known contact with chromium at home or at work. The only source of chromium we found for these three cases was the radiopaque substances used to visualise or enhance the image of the brain or cord. Case 1 also received KCL solution and Mylanta that is $3 \mathrm{mg}$ chromium (during the period of 1 month) plus $52 \mathrm{ml}$ of Pantopaque (45 $\mu \mathrm{g}$ chromium) over the space of three days plus $12 \mathrm{ml}$ Conray $(2.4 \mu \mathrm{g}$ of chromium), approximately during the same period of time. Case 3 with malignant astrocytoma received 18 $\mathrm{ml}$ of Hypaque (3.6 $\mu \mathrm{g}$ chromium IV); plus $12 \mathrm{ml}$ Conray $(2 \cdot 4 \mu \mathrm{g} \mathrm{IV})$. Case 2 received a similar amount of Conray the day before her death.

These quantities are very small compared to the figures for chromium toxicity in animals. The LD50 (lethal dose for $50 \%$ of the animals intoxicated) for trivalent or hexavalent chromium salts (as the metal) for rats and mice varies from 10 to $23 \mathrm{ppm}(\mathrm{mg} / \mathrm{kg})$. The MLD (maximum lethal dose) for IV trivalent or hexavalent chromium salts (as the metal) in rats, mice, dogs and rabbits varies from $7 \cdot 2$ (sodium dichromate) to 520 (chromic acetate) ppm. The figures for toxicity of chromium to humans are not known. Figures for air pollution of chromium to humans are known. ${ }^{2}$

There is little information concerning the neu rotoxicity of chromium in humans. Lehmann and $\mathrm{Ule}^{12}$ have shown that chromic sutures implanted in the trigeminal nerve for 3 weeks cause circumscribed inflammation and demyelination, axonal swelling and proliferation of Schwann cells. This model for demye lination was the basis of physiological studies of abnormal repetitive action potential generations bys Burchiel. ${ }^{13}$ Perbellini and DeGrandi ${ }^{14}$ reported a case with a vasculopathy and neuropathy localised to the upper limb in a worker exposed to nickel and chromium. Tandon et $a^{15}$ studied the distribution of chromium in poisoned rats and noticed that it was elevated in the brain when compared to other organs. (3) The neurotoxicity of radiological contrast agents Neurological effects are among the most important complications of IV administration of radiological contrast agents such as iothalamate and diatrizoate meglemine (Conray, Hypaque). These include seizures, stroke, cerebral oedema. The toxicity is attributable to the iodine-containing anions but the choice of cation can modify the toxicity. Iophendylate (Pantopaque) administered intrathecally may cause acute or chronic meningeal reactions. There are no reports in the literature of contrast agents causing the neuropathology seen in our three cases.

In conclusion, it is demonstrated in this report that chromium, a highly toxic element can enter the human brain via the pathological pallidal blood vessels showing vascular siderosis. We do not have firm evidence that chromium caused the pathology in 
these three cases, although the clinical and pathological findings in case 1 suggest that chromium may have contributed to the pathology.

The study of these findings suggests that care should be taken in the use of radiopaque substances in patients with evidence of vascular pathology, such as pallidal calcifications, which are manifestations of vascular siderosis, visible on plain radiographs. These findings agree with the view that a breakdown in the blood-barrier caused by neuropathology in humans, facilitates the entry into the brain of radiological contrast agents and subsequent neurological effects. ${ }^{16}$

This study was funded by the Centre National de la Recherche Scientifique (France), Institut National de la Sante et de la Recherche Scientifique (SG27), General Research Funds from Jefferson Medical College. I thank my colleagues for their support and assistance, Drs Pierre Galle, Richard Berry, RE Scourolles, JF Hauw and Mr RG White, from the Universite de Paris, Jefferson Medical College and the University of Pennsylvania-LRSM.

\section{References}

${ }^{1}$ Duckett S, Galle P. Electron Microprobe Analysis of Normal and Pathological Neural Tissue with Wavelength Dispersive X-Ray Spectrometry. In: Harris J, ed. Metal Ions in Neurology and Psychiatry. New York: Alan J Liss, 1985.

${ }^{2}$ Langard S, Norseth T. Chromium. In: Friberg L, Nordberg GF, Vouk VB, eds. Handbook on the Toxicology of Metals. New York: Elsevier 1979:387-97.
${ }^{3}$ Duckett S, Galle P, Kradin R. The relationship of Parkinson's disease and vascular siderosis. Ann Neurol 1977;2:225-30.

${ }^{4}$ Alexander L, Putnam TJ. Pathological alterations of cerebral vascular patterns. Res Publ Assoc Res Nerv Ment Dis 1938;18:471-543.

${ }^{5}$ Denny-Brown D. The Basal Ganglia. Oxford: Oxford University Press, 1962:50.

${ }^{6}$ Mertz W. Chromium and its relation to carbohydrate metabolism. In: Trace Elements. Medical Clinics of North America. WB Saunders, 1976;60(4):739-44.

${ }^{7}$ Chromium. Pub National Academy of Sciences. Washington DC, 1974.

${ }^{8}$ Occupational Exposure to Chromic Acid. Pub US-NIOSH, 1973.

9 Jeejeebhoy KN, Chu RC, Marliss EB, Greenberg GR, Bruce-Robertson A. Chromium deficiency, glucose intolerance and neuropathy reversed by chromium supplementation in a patient receiving long term parenteral nutrition. Am J Clin Nut 1977;30:531-8.

${ }^{10}$ Anspaugh LR, Robison WL, Martin WH, Lowe OA. Compilation of Published Information on Elemental Concentrations in Human Organs in both Normal and Disease States. Lawrence Livermore Laboratory, University of California Livermore, 1971.

${ }^{11}$ Goody W, Hamilton EI, Williams TR. Spark-source mass spectrometry in the investigation of neurological disease. Brain 1975;98:65-70.

${ }^{12}$ Lehmann HJ, Ule G. Electrophysiological findings and structural changes in circumscript inflammation of peripheral nerves. Prog Brain Res 1964;6:169-73.

13 Burchiel KJ. Ectopic impulse generation in demyelinated axons: effects of $\mathrm{Pa} / \mathrm{CO} 2, \mathrm{pH}$ and disodium edetate. Ann Neurol 1981;9(4):378-83.

14 Perbellini L, DeGrandi D. Neuro-arteropatia Verosimilimente correlata ad esposizione a nickel e cromo. Med Lavoro 1979;4:318-22.

15 Tandon SK, Bebari JR, Kochru DN. Distribution of chromium in poisoned rats. Toxicol 1979;13:29-34.

16 Junck L, Marshall WH. Neurotoxicity of radiological contrast agents. Ann Neurol 1983;13(5):469-84. 